\title{
Neuroendoscopic lavage and continuous drainage of ventricles: Treatment of pyocephalus in a newborn
}

\author{
Ram Babu Joshi, MBBS, Rupendra Bahadur Adhikari, MD, PhD; Amit Thapa, MS, MCh \\ Department of Neurosurgery, Grande International Hospital, Kathmandu, Nepal
}

\section{Corresponding author}

Ram Babu Joshi, MBBS

Email: rambabu.joshi@yahoo.com

Received 2 Dec 2018

Accepted 19 Dec 2018

\section{ABSTRACT}

Pyocephalus/Cerebral ventricular empyema is a serious life threatening complication of acute pyogenic meningitis. The primary treatment of ventriculitis is administration of antibiotics. With recent advances, neuroendoscopic lavage (NEL) of ventricles through direct visualization has helped save lives when multipronged approaches including intravenous (IV) antibiotics, intrathecal antibiotics and continuous drainage of cerebrospinal fluid (CSF) fails.

We report a case of a 23-day old neonate who developed pyocephalus as a complication of pyogenic meningitis. He did not respond favorably to initial IV antibiotic treatment for two weeks. NEL of ventricles was performed. Thick pus/flakes inside the ventricles had caused obstructive hydrocephalus. Continuous CSF drainage was done through strategically placed multiple external ventricular drains. Interval ventriculo-peritoneal shunt was done bilaterally after the CSF was macroscopically/ microscopically clear of visible debris, and sterile. Microbiological and clinical cure was achieved and the child survived and is thriving well at last follow-up at the age of 6 months.

Key words: Neuroendoscopic lavage, pyocephalus, newborn, ventriculitis, ventricular empyema

\section{Introduction}

Pyocephalus, also known as cerebral ventricular empyema, a rare form of pyogenic ventriculitis is the most severe complication of acute pyogenic meningitis (APM) occurring in $30 \%$ of all patients and $90 \%$ of neonates ${ }^{1}$. Primary treatment of meningitis is the use of appropriate antibiotics but has limited efficacy due to the blood brain barrier². Other treatment options like intraventricular (IV) irrigation and intrathecal (IT) injection of antibiotics have a limited role owing to complications related to the invasive nature of the procedures, and neurotoxicity sequelae. Neuroendoscopic treatment of ventricular infections usually requires multi-staged surgical approach. The clinical outcome in the present case is significantly better than that treated with systemic and intraventricular antibiotics reported in the literature.

\section{Case report}

A 23-day old male baby delivered at 37 weeks of gestation via Caesarean section was admitted for respiratory distress syndrome with congenital pneumonia with Klebsiella $s p$, and treated with 2 weeks of intravenous (IV) antibiotics. He was re-admitted 5 days later to the NICU after he developed high fever. There was no history of seizure. The neonate was lethargic and vacant staring was present. CSF picture was consistent with bacterial meningitis. Ultrasound (USG) of the cranium showed basal exudates and heterogeneous echotexture of bilateral frontal lobe at peri interhemispheric falx region, suggestive of meningitis. Blood culture yielded Klebsiella sp., CSF was sterile.

The neonate developed multiple episodes of seizures. Repeat USG cranium showed mild hydrocephalous. Despite antibiotic upgrade, the neonate remained febrile, anterior fontanel was bulging and he developed opisthotonus posture. USG cranium was suggestive of brain abscess, which was confirmed by MRI (Fig. 1). He underwent left frontal extra ventricular drain (EVD) placement. Thick brownish CSF with sediments came out through the EVD. 


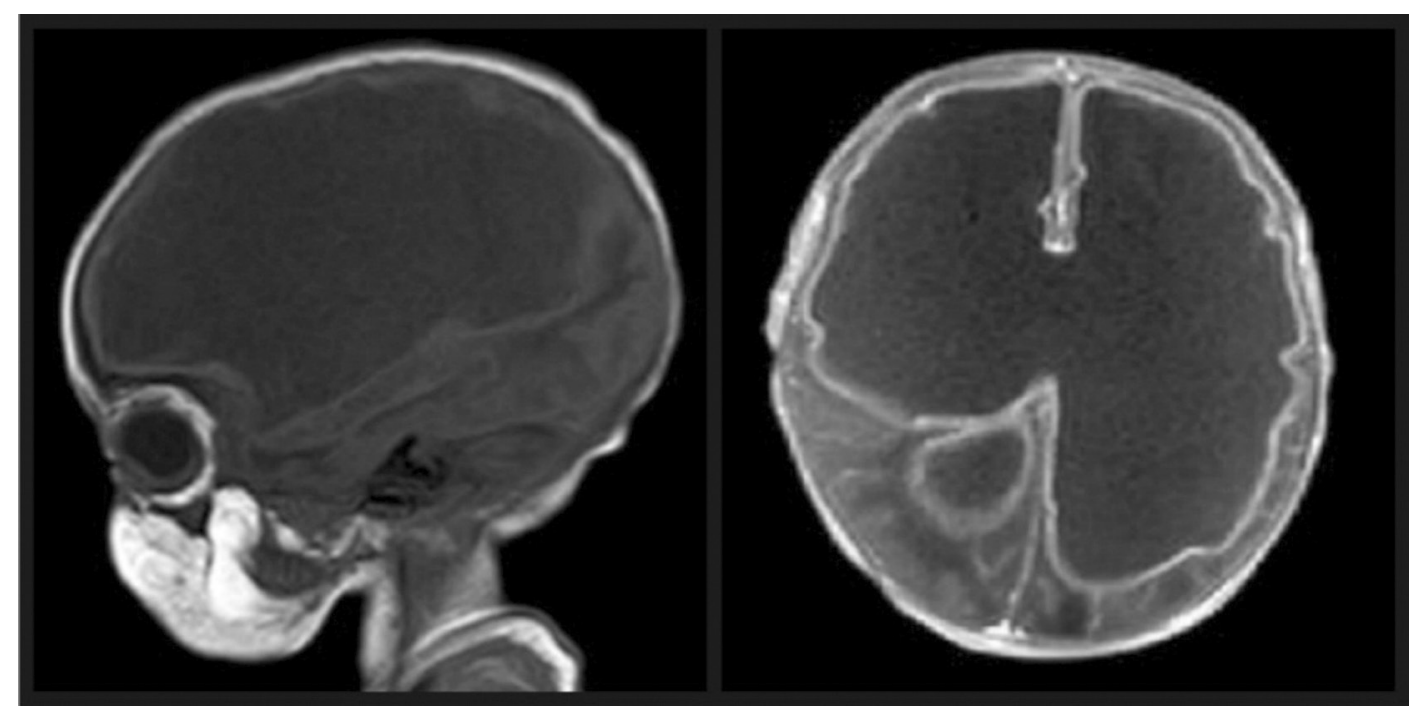

Figure 1: Sagittal MRI / Axial CEMRI head - Grossly dilated lateral ventricles noted. Debris in posterior dependent parts giving fluid debris level. Enhancement of ventricular wall is seen in post contrast study. Marked thinning of brain parenchyma noted predominantly in frontal lobes. Features consistent with pyocephalus.

Neuroendoscopic evaluation of ventricles and lavage was performed following a repeat $\mathrm{CT}$ after a week (Fig.2).

Plenty of cotton wool like pus/flakes could be seen attached to the walls of lateral ventricles. Detailed anatomy of lateral ventricle, choroid plexus

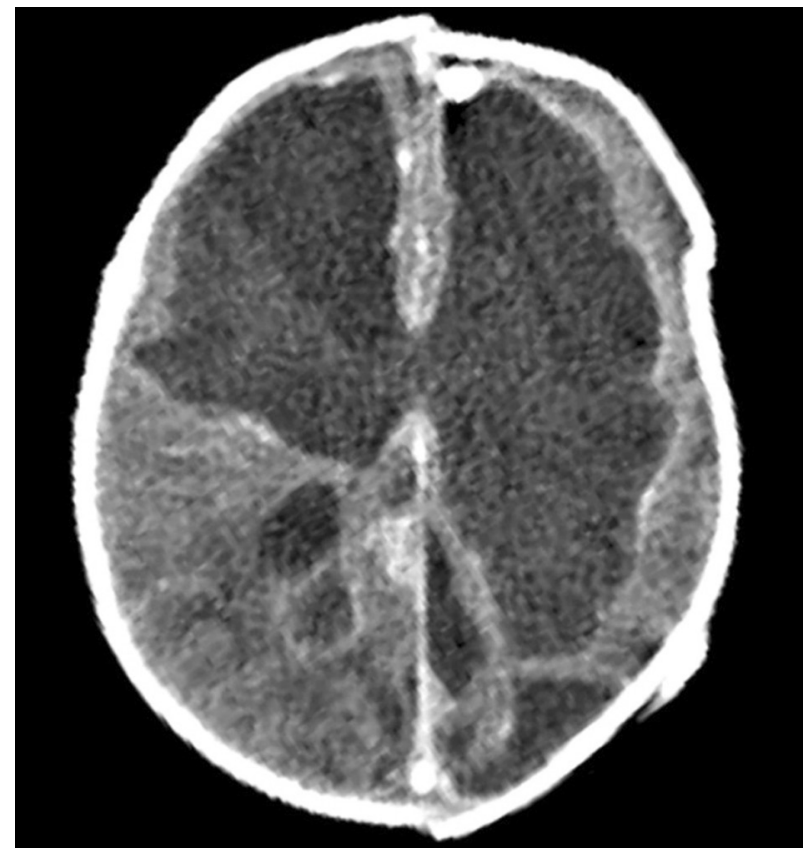

Figure 2: Axial CECT head - Gross dilatation of bilateral lateral ventricles Thickening of the ependymal lining of the ventricles is seen which shows moderate enhancement in post contrast study. Marked compression with thinning of brain parenchyma is seen in bilateral frontal and left parietal lobe. Drain tube is seen in situ. was obscured by the CSF pus/flakes. Thorough ventricular lavage was done. Since the lateral ventricles were not communicating, 2 EVDs were placed with long tunneling to allow for CSF drainage/ lavage for 3-week duration. CSF for culture was sent on every third day, all of which were reported sterile. Repeat EVD was placed through Keen's point bilaterally and inside the encapsulated right frontal abscess under USG guidance and kept for 2 weeks.

MRI head (Fig. 3) was repeated that confirmed abating of the ventriculitis, following which he underwent bilateral ventriculo-peritoneal shunting at Frazier's point bilaterally. In total, he received 6 weeks of IV antibiotics. At one-month, and subsequent follow-ups, the child was active, feeding well and thriving. His CT head showed decreased in the size of hydrocephalus, and gain in brain parenchymal volume (Fig. 4).

\section{Discussion}

Ventriculitis is frequently present with meningitis, as result of a cerebral abscess communicating with the ventricles, or may be a complication of shunt ${ }^{3}$. Most literatures report pyocephalus as a sequelae of cerebral abscess. Foraminal attachments in ventriculitis causes obstruction that isolates the ventricles which then fills up with pus - Pyocephalus ${ }^{3}$. The formation of fibrous capsules in neonates is slower than in older children. This delayed encapsulation ensues spill-over of the pus into the ventricles causing pyocephalus ${ }^{4}$. 


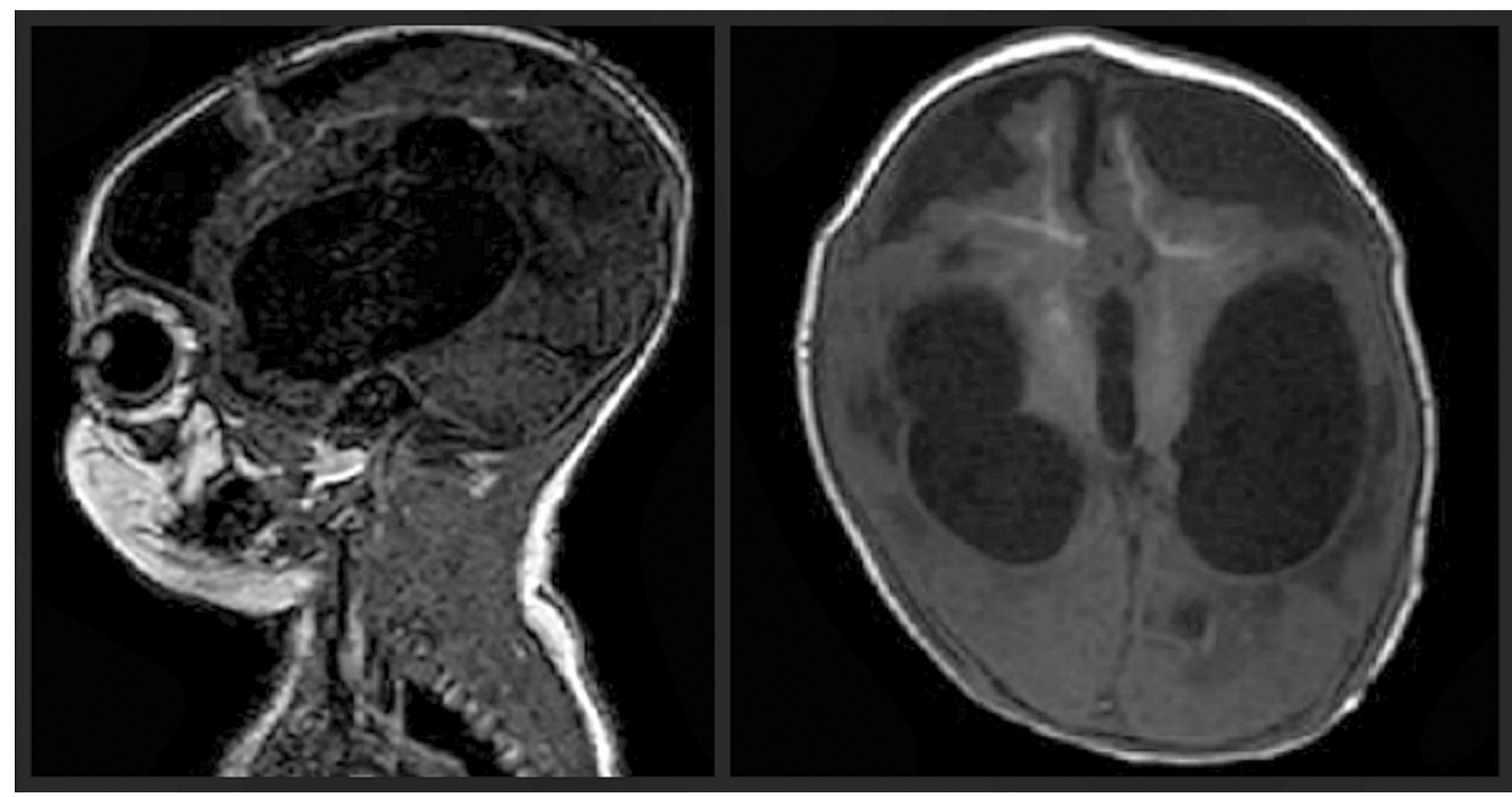

Figure 3: Sagittal/ Axial MRI head - Markedly dilated temporal horns of lateral ventricles. Frontal horns are not dilated. Left occipital horn is also slightly prominent. Third ventricle is dilated. Fluid-debris differential attenuation has disappeared compared to Fig.1 and Fig.2.

Although antibiotics are primary treatment of pyocephalus, multiple factors like emergence of multidrug resistance strain of gram negative bacteria, poor CNS penetration of IV antibiotics, and impaired drainage of infected CSF result in

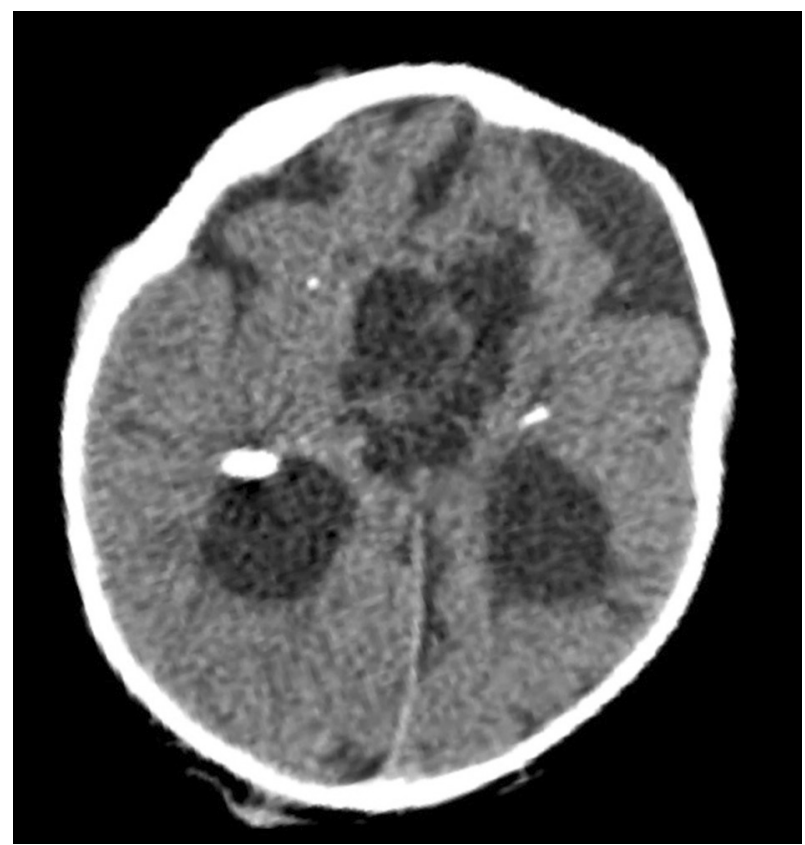

Figure 4: NCCT axial head at one month after VP shunting - Moderate dilatation of bilateral lateral and third ventricles, left frontal minimal subdural effusion seen - hydrocephalus ex-vacuo. Ventricular tips of VP shunt are seen on both sides. Compared to past scans, brain parenchymal volume has increased. antibiotic unresponsiveness ${ }^{5}$. In such setting, NEL coupled with continuous CSF drainage system provides a conduit for clearing up the debris, and natural lavage. Many articles have shown higher rate of survival after NEL over antibiotic treatment alone for pyocephalus ${ }^{2,5-9}$. Schulz et al. described their experience in using NEL in 14 neonates. Among them 3 underwent NEL for antibiotic unresponsive ventriculitis. They found turbid CSF with pus/flakes attached to the ventricular wall intraoperatively similar to our case ${ }^{10}$.

NEL is a safe and effective treatment for pyocephalus in children with infectious conditions. It allows better visualization so that the pus/flakes can be removed $^{2}$. NEL results in a decreased number of overall shunt revisions in shunt-dependent patients. NEL favorably impacts to decrease the number of recurrent infections as it allows efficient removal of ventricular sediment, tissue dross as well as sludged blood from hard- to-approach areas like third ventricle and temporal and occipital horns of lateral ventricle. It also allows for manual recanalization of both inflammatory septum and the atresia of inter- ventricular foramen or midbrain aqueduct, thus improving the CSF circulation and facilitating better irrigation of antibiotic saline ${ }^{2,6}$. NEL stabilizes the clinical condition in neonates and buys time for further growth and increase in body 


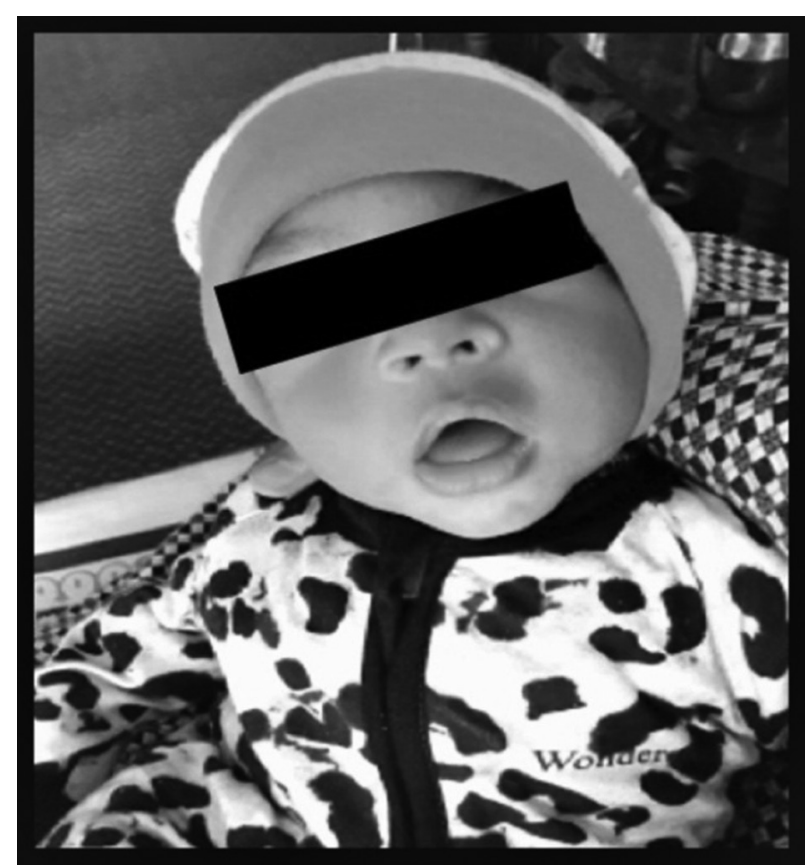

Figure 5: Child at 6 months of age

weight in order to gain better precondition for VP shunting as to say it has the secondary benefit to alleviate further VP shunt treatment ${ }^{7,11}$.

Historically, Youl published the earliest case of cerebral abscess in 8-month old boy in $1847 \mathrm{AD}^{4}$. Three ounces of pus from each lateral ventricle at autopsy in a 3-month old child described by Hughes-will-shire is the first account of pyocephalus in literature ${ }^{4}$. After studying the role of NEL in multidrug resistance gram negative bacteria in 7 patients, Kumar et al. concluded that NEL should be considered in any patient with ventriculitis if infection persists even after 7-10 days of IV and IT antibiotics 5 . Some authors have reported using NEL only after 2 weeks of antibiotics treatment. Terada et al. retrospectively analyzed 14 patients, 6 of whom were treated with NEL and rest with conventional treatment. They concluded that there is high efficacy of earlier intervention with NEL as soon as patient is diagnosed with ventriculitis?.

Before the dawn of antibiotic era, the mortality rate of pyocephalus was $100 \%$. Use of antibiotics has helped reduce the mortality ranging from 30 to $80 \%{ }^{7}$. In patient with multidrug resistance gram negative ventriculitis, around $71 \%$ of mortality has been reported ${ }^{5}$. There is a very good outcome of patient with pyocephalus after NEL treatment as seen in the cases discussed in the articles published. Patient is notified microbiologically cured when three consecutive samples of CSF culture is sterile. Similarly, clinical cure can only be achieved if patient has no evidence of central nervous system infection at the time of discharge ${ }^{5}$.

\section{Conclusion}

Neuroendoscopic lavage with continuous CSF drainage is an effective, and rational treatment strategy in neonatal pyocephalus. Six weeks of IV antibiotics cover is advisable even after repeated CSF studies are microbiologically sterile. Anatomical aberrations and fluid dynamic changes in the setting of ventriculitis warrants ventriculoperitoneal shunting at the earliest.

\section{References}

1. Barloon TJ, Yuh W, Knepper LE, Biller J, Ryals TJ, Sato Y. Cerebral ventriculitis: MR findings. J Comput Assist Tomogr. 1990;14(2):272-5.

2. Hu Z-Q, Guan F, Huang H, Ren Z-Y, Wang Z-Y, Fu J-D, et al. A multi-center study of neuro-endoscopic treatment for ventricular infection. Int J Clin Exp Med 2018;11(3):2398-405.

3. Kuzeyli K, Aktürk F, Çakir E, Baykal S, Karakus M, Duru S. Ventricular empyema and pyocephalus: A case report. Turkish Neurosurgery.6(1-2). Turk Neurosurg. 1996;6:25-7.

4. Izquierdo JM, Sanz F, Coca JM, Vila F, Dierssen G. Pyocephalus of the newborn child. Childs Brain 1978;4:161-9.

5. Kumar A, Agrawal D, Sharma BS. The role of endoscopic lavage in recalcitrant multidrug-resistant gram-negative ventriculitis among neurosurgical patients. World Neurosurg. 2016;93:315-23.

6. Gaderer C, Schaumann A, Schulz M, Thomale UW. Neuroendoscopic lavage for the treatment of CSF infection with hydrocephalus in children. Childs Nerv Syst. 2018;34(10):1893-903.

7. Terada $Y$, Mineharu $Y$, Arakawa $Y$, Funaki T, Tanji M, Miyamoto S. Effectiveness of neuroendoscopic ventricular irrigation for ventriculitis. Clin Neurol Neurosurg. 2016;146:147-51.

8. Lang M, Habboub G, Moore NZ, Recinos VMR, Mohammadi AM, Nagel S, et al. Neuroendoscopic evacuation of intraventricular empyema using a side-cutting aspiration device. J Clin Neurosci. 2018;47:323-7.

9. Wang F, Yao XY, Zou Z-R, Yu H-L, Sun T. Management of pyogenic cerebral ventriculitis by neuroendoscopic surgery. World neurosurg. 2017;98:6-13. 
10. Schulz M, Bührer C, Spors B, Haberl H, Thomale UW. Endoscopic neurosurgery in preterm and term newborn infants - a feasibility report. Childs Nerv Syst. 2013;29(5):771-9.

11. d'Arcangues C, Schulz M, Bührer C, Thome U, Krause $\mathrm{M}$, Thomale U-W. Extended experience with neuroendoscopic lavage for posthemorrhagic hydrocephalus in neonates. World Neurosurg. 2018;116:e217-e224.

12. Chaiyakulsil C, Prommalikit O. Successful medical treatment in a child with E. coli ESBL meningitis with acute communicating hydrocephalus and ventricular empyema: a case report. J Med Assoc Thai. 2012;95 Suppl 12:S138-41.
13. Schultz P, Leeds NE. Intraventricular septations complicating neonatal meningitis. J Neurosurg. 1973;38(5):620-6.

14. Bakshi R, Kinkel PR, Mechtler LL, Bates VE. Cerebral ventricular empyema associated with severe adult pyogenic meningitis: computed tomography findings. Clin Neurol Neurosurg. 1997;99(4):252-5.

15. Schulz $M$, Bührer $C$, Pohl-Schickinger $A$, Haberl $\mathrm{H}$, Thomale UW. Neuroendoscopic lavage for the treatment of intraventricular hemorrhage and hydrocephalus in neonates. J Neurosurg Pediatr. 2014;13(6):626-35. 